Actions to improve documented pain assessment in adult patients with injury to the upper extremities at the Emergency Department A cross-sectional study

\title{
Sturesson, L.
}

2016-03

Sturesson, L, Lindstrom , V , Castren , M , Niemi-Murola, L \& Falk , A -C 2016 , ' Actions to improve documented pain assessment in adult patients with injury to the upper extremities at the Emergency Department A cross-sectional study ' , International Emergency Nursing , vol. 25 , pp. 3-6 . https://doi.org/10.1016/j.ienj.2015.06.006

http://hdl.handle.net/10138/223936

https://doi.org/10.1016/j.ienj.2015.06.006

publishedVersion

Downloaded from Helda, University of Helsinki institutional repository.

This is an electronic reprint of the original article.

This reprint may differ from the original in pagination and typographic detail.

Please cite the original version. 


\title{
Actions to improve documented pain assessment in adult patients with injury to the upper extremities at the Emergency Department - A cross-sectional study
}

\author{
L. Sturesson RN ${ }^{a, *}$, V. Lindström PhD, RN (Coordinator Academic EMS) ${ }^{b, c}$, \\ M. Castrén MD, PhD (Professor) ${ }^{\mathrm{a}, \mathrm{d}}$, L. Niemi-Murola MD, PhD (Researcher) ${ }^{\mathrm{b}, \mathrm{e}}$, \\ A.-C. Falk RN, CCRN, PhD f,g \\ a Department of Clinical Science and Education, Section of Emergency Medicine, Karolinska Institutet, Södersjukhuset, Stockholm, Sweden \\ ${ }^{\mathrm{b}}$ Department of Clinical Science and Education, Karolinska Institutet, Södersjukhuset, Stockholm, Sweden \\ ${ }^{\mathrm{c}}$ Academic EMS, Stockholm, Sweden \\ ${ }^{\mathrm{d}}$ HUCH Emergency Care, Helsinki University Hospital, Helsinki University, Finland \\ e Department of Anaesthesia and Intensive Care Medicine, Helsinki University Hospital, Finland \\ ${ }^{\mathrm{f}}$ Department of Neurobiology, Care Sciences and Society, Division of Nursing, Karolinska Institutet, Sweden \\ ${ }^{\mathrm{g}}$ Intensive Care Unit, Karolinska University Hospital, Solna, Sweden
}

\section{A R T I C L E I N F O}

\section{Article history:}

Received 12 February 2015

Received in revised form 16 June 2015

Accepted 17 June 2015

\section{Keywords:}

Emergency care

Acute pain

Pain management

Pain assessments

Guidelines

Evidence based care

Documentation

\begin{abstract}
A B S T R A C T
Background: Pain is one of the most common symptoms in the Emergency Department (ED) and is the cause of more than half of the visits to the ED. Several attempts to improve pain management have been done by using, for example, standards/guidelines and education. To our knowledge no one has investigated if and how different actions over a longitudinal period affect the frequency of pain documentation in the ED. Therefore the aim of this study was to describe the frequency of documented pain assessments in the ED.

Method: A cross-sectional study during 2006-2012 was conducted. The care of patients with wrist/arm fractures or soft tissue injuries on upper extremities was evaluated.

Result: Despite various actions our result shows that mandatory pain assessment in the patient's computerized medical record was the only successful intervention to improve the frequencies of documentation of pain assessment during care in the ED. During the study period, no documentation of reassessment of pain was found despite the fact that all patients received pain medication.

Conclusion: To succeed in increasing the frequency of documented pain assessment, mandatory pain rating is a successful action. However, the re-evaluation of documented pain assessment was nonexisting.
\end{abstract}

(C) 2016 Elsevier Ltd. All rights reserved.

\section{Introduction}

Pain is one of the most common symptoms treated in the emergency department (ED) and is the cause of more than half of the visits to the ED (Cordell et al., 2002; Wilson and Pendleton, 1989). Previous studies have concluded that pain management is inadequate and oligoanalgesia is reported to be a problem in the ED (Fosnocht et al., 2005; Niruban et al., 2010; Todd et al., 2007). Barriers to achieving adequate pain relief for patients in the ED have been shown to be: failure to assess and acknowledge pain, lack of

\footnotetext{
* Corresponding author. Department of Clinical Science and Education and Section of Emergency Medicine, Karolinska Institutet, Akutmottagningen, Södersjukhuset AB, Sjukhusbacken 6, S - 11883 Stockholm, Sweden. Tel.: +4686162107; fax: +4686161333

E-mail address: lars.sturesson@sodersjukhuset.se (L. Sturesson).
}

guidelines, failure to document pain, failure to assess treatment correctly, attitudes and inadequate knowledge among personnel (Motov and Khan, 2008). Since oligoanalgesia may be associated with increased risk of complications such as sleep disturbance, delirium, depression, and decreased response to interventions for other illnesses, especially among the elderly (Abou-Setta et al., 2011), the patient's pain should be considered as the fifth vital sign (Purser et al., 2014). Several attempts to improve pain management in the ED have been made by using guidelines (Eder et al., 2003), implementing assessment using pain scales (Ritsema et al., 2007), and by education of the personnel (Decosterd et al., 2007). Documentation of pain assessment conducted in the ED has been shown to have an effect on pain management (Vazirani and Knott, 2012), but there is a large variation, with a range of 57-94 per cent in the frequency of documented pain assessment (Brown et al., 2003; Eder et al., 2003; Purser et al., 2014). In Sweden there are no national regulatory requirements for pain management in the ED, but local 
guidelines concerning pain management have been developed. All the above described attempts have, to some extent, improved pain management in the ED, but pain management is still reported to be insufficient (Lewén et al., 2010). To our knowledge, no one has investigated if and how different interventions over a long period affect the frequency of pain documentation in the ED. Therefore, the aim of this study was to describe the frequency of documented pain assessments in the emergency department.

\section{Material and methods}

A cross-sectional study of medical records was conducted from 2006 to 2012. The study was approved by the Medical Research Ethics Committee, Stockholm, Sweden (2008/991-31/3).

\subsection{Setting}

The study was conducted on patients with wrist/arm fractures or soft tissue injuries in a 24-hour adult ED ( $>15$ years) at a public urban teaching hospital in Stockholm. Each year, the hospital receives approximately 7300 patients with acute life-threatening situation/condition by ambulance. In the health care system in Stockholm, patients with orthopaedic and/or surgical injuries $>15$ years are always treated at an adult ED. During the study period the number of patients attending the ED increased from 79,277 in 2006 to 109,982 in 2012 without the ED being expanded. Moreover, the patients with wrist/arm fractures or soft tissue injuries increased from 1353 to 1657 . The hospital beds decreased from 560 to 505 over the same period. The ED serves all adult patients with surgical cardiology, orthopaedics and internal medicine requirements, and no referral is needed. The ED has transfer agreements for patients requiring more comprehensive care such as neurosurgery and burn injuries. The personnel working with direct patient care in the ED are: physicians specializing in cardiology, surgery, orthopaedics, internal medicine or emergency medicine; and registered nurses (RN) and RN with additional training in emergency care. There are approximately $250 \mathrm{RN}$ and nurse assistants working at the ED. The RNs perform the first triage, supported by standardized protocols. The aim of this first triage is to identify the patients' need of care based on the acuity of their condition. During the study period, patients with wrist/arm fractures or soft tissue injuries were treated by orthopaedic surgeons or emergency physicians, and by nurses with or without additional emergency care training.

\subsection{Data collection and variables}

Patients' medical records were used for data collection. To assess the frequencies of pain rating over time, data were collected from patients' medical records. Inclusion criteria were: The first ten medical records in the admission system for the ED of adult patients ( $>15$-years or older) every month $2006-2012$ (=120 patients/ year) with wrist/arm fractures or soft tissue injuries on the upper extremities. Since we wanted to investigate the frequency of documentation regarding pain assessment we included patients that had received analgesics. Patients with wrist/arm fractures or soft tissue injuries on the upper extremities were selected since they are a common group of patients and, among fractures, wrist fractures are the most frequently treated in the ED (Court-Brown and Caesar, 2006). Such patients can also be easily identified by the triage nurse (an RN with additional training in emergency care). Also, in accordance with local guidelines implemented in year 2002, the RN is able to administer oral and/or intravenous pain medication at admission to this group of patients by using the nurse-initiated analgesic protocols. The protocol states that the RN should assess the patients' pain using a pain rating scale (NRS), including documentation of pain assessment before and after administering analgesics. The authors reviewed the patients' medical record and collected variables of documented pain ratings according to NRS, reassessment of pain rating (yes or no), and demographic characteristics such as gender and age. All collected variables were manually entered into excel. No data were missing.

\subsection{Interventions to improve assessment and documentation of pain}

The interventions aiming to improve the pain assessment and documentation in the ED during the study period were as follows:

- In 2002 a guideline describing a pain policy programme was published and implemented in the ED. Overall, the policy programme clearly stated that all patients with pain should have their pain assessed and documented. The only implementation of this guideline was hospital management giving written information to the ED personnel that a new pain assessment guideline was in place.

- In 2006 Stockholm County Council (SCC) published a report on pain (Stockholm County Council 2006). The aim of the report was to get health care personnel focused on pain and its treatment. The report was available to all the personnel in the ED.

- In September 2007 a group of personnel with a special interest in and knowledge of pain was formed by the first author (LS) in the ED. The group consisted of six RNs and nurse assistants all working in the ED. The overall purpose of the group was to educate and facilitate other personnel in the ED in increasing pain assessment and documentation. The group communicated their message through various types of staff meetings.

- At the beginning of 2008, the heads of personnel in the ED decided that the Numeric Rating Scale (NRS) should be used as the pain rating scale since it is a validated pain rating scale for use in the ED (Berthier et al., 1998; Bijur et al., 2003). In addition to the introduction of, and education about, the NRS the RNs were instructed to note the NRS score in the patient's medical record when the patient arrived at the ED.

- In May 2008, to make pain management more visible for the RNs, a reminder of pain assessment and the use of the NRS were noted in the patients' medical records.

- In January 2009, aiming to remind the RNs to assess and document pain, the patients were informed by the triage nurse about the pain rating. The information was meant to be a reminder to the RNs to perform and document pain assessment.

- In October 2010, the patient medical report was computerized and pain assessment became mandatory.

\subsection{Data analysis}

Data analysis was performed using Microsoft Office, Excel 2007. The results are presented using descriptive statistics (numbers and per cent).

\section{Results}

During the study period a total of 840 (120/year) patients with wrist/arm fractures or soft tissue injuries and who had received analgesics at the ED were included. The medical records were examined regarding documentation of pain assessment and reassessment postanalgesia. Patients included in the study were predominantly older females (Table 1).

During 2006, the guidelines concerning assessment and documentation of pain were implemented in the ED, but adherence to guidelines was poor, as shown in Table 2. In 2007 a group of personnel with special interest in pain was put together and the frequency of documented assessed pain increased for a short period. Despite various actions, there was no sustained effect until the documentation was mandatory and computerized during 2010, as 
Table 1

Characteristics of ED patients with injury to the upper extremities $(n=840)$.

\begin{tabular}{llll}
\hline & & Male & Female \\
\hline Participants (\%) & & $287(34)$ & $553(66)$ \\
Age & median & $35.5(15-95)$ & $61(15-98)$ \\
& mean \pm SD & $38.7 \pm 19$ & $60.2 \pm 19$ \\
\hline
\end{tabular}

Table 2

Frequency of documented pain assessments 2006-2012.

\begin{tabular}{lllllllc}
\hline & 2006 & 2007 & 2008 & 2009 & 2010 & 2011 & 2012 \\
\hline Jan & - & - & - & $c 1$ & 2 & 9 & 8 \\
Feb & - & - & 3 & 4 & 2 & 9 & 8 \\
Mar & - & - & 3 & 5 & 3 & 9 & 9 \\
Apr & - & - & 3 & 2 & 3 & 9 & 10 \\
May & - & - & b2 & 1 & 4 & 9 & 10 \\
Jun & 1 & - & 2 & 1 & 5 & 10 & 8 \\
Jul & - & - & 1 & 2 & 3 & 7 & 7 \\
Aug & - & - & 2 & 4 & 1 & 7 & 7 \\
Sep & - & 7 & 1 & 1 & 2 & 9 & 8 \\
Oct & - & 1 & 2 & 1 & $\mathrm{~d} 8$ & 8 & 8 \\
Nov & - & 7 & 3 & 3 & 9 & 10 & 7 \\
Dec & - & 4 & 2 & 3 & 8 & 10 & 6 \\
Frequency & $1 / 120$ & $19 / 120$ & $27 / 120$ & $29 / 120$ & $50 / 120$ & $115 / 120$ & $96 / 120$
\end{tabular}

a Group with aim of educating and facilitating other personnel in the ED started.

b Reminder of pain assessment in medical record.

c Visual Analogue Scale (VAS) distributed to every patient admitted to ED.

d Pain assessment became mandatory and computerized.

shown in Table 2. During the study period, no documentation of reassessment of pain was found, despite the fact that all patients received pain medication.

\section{Discussion}

The aim of this study was to describe the frequency of documented pain assessments in an ED. Our result shows that mandatory pain assessment in the patient's computerized medical record was successful as an intervention to improve the frequency of documented pain assessment in the ED. The result was maintained for 21 months, before a slight decline in the frequency of pain assessment was shown. This result is consistent with the result of Vazirani and Knott concerning the long-term effect of different interventions to improve pain assessment (Vazirani and Knott, 2012). However, our result shows a sustainable effect for a longer period of time than ever shown before. The decrease in documented pain assessment in 2012 may indicate that the RNs have found a way to 'work-around' the mandatory documentation. Instead of assessing and documenting the patient's pain in accordance with NRS the RNs document "pain not possible to assess". However, despite the fact that all patients in our study received pain medication, no reevaluation of the patient's pain was found to be documented in the medical records. As examples of interventions that could improve pain documentation in the future, one could look at developments such as mandatory documentation of re-evaluation, or letting the patients measure their own pain intensity in the medical record.

As reported by Sampson et al. (2014), studies should include patient-centred outcomes such as reduction in pain score or better patient satisfaction as improved criteria of pain management. Our result shows that guidelines and educational efforts did not have any effect on the pain assessment frequencies, which differs from Decosterd et al. (2007), whose result showed an increase of pain assessment frequencies. Whether or not this depends on the kind of educational effort that has been made or how the guidelines have been created would be interesting to investigate further. Since pain is a frequent symptom reported by patients who visit the ED (Cordell et al., 2002; Wilson and Pendleton, 1989) it is remarkable that there was such a low frequency of documented pain assessment (NRS) during the first years of the study period, despite the fact that the patients had received initial pain medication. In contrast to other studies (Gordon et al., 2008), our result shows a disappointing rate of documentation of pain assessment despite the fact that the hospital already had a guideline stating that pain assessment was mandatory when the patient reported any pain. Other results show that when pain assessment is low, the treatment of pain is lacking (Silka et al., 2004) but this was not shown in our study. However, this is a question of patient quality within the health care system and should be investigated further.

The SCC report published in 2006 (County Council Stockholm, 2006) focused on acute postoperative pain and not acute pain reported in the ED, similar to Sampson et al. (2014). This could have had an impact on the ED personnel's readiness to accept the guidelines since the context was different, as was the patients' description of the pain. An acute visit to an ED can be associated with anxiety, fear and depression, which may not always be experienced at a planned surgery, where the patient and the medical professionals have been able to prepare for the situation.

It is also known that anxiety and fear can heighten the experience of pain intensity (Tanasale et al., 2013). Therefore, acute pain in the ED should be further investigated both from the patient's and the ED personnel's perspectives. The group that intended to educate other personnel in the ED started in 2007 and had a positive impact on pain assessment. The personnel who created the group also acted as facilitators, as described by Berben et al. (2012). Despite these interventions in the ED, personnel may not have recognized pain as the fifth vital sign, as described by Purser et al. (2014). Whether or not this could be improved by educational interventions is yet to be investigated.

\subsection{Limitations}

There are several limitations to this study. The documentation of pain assessment was studied and not the actual assessment of patients' pain. Also, it is not known to what extent triage nurses administer analgesic prior to the physicians' examination of the patient. Excluding patients not receiving analgesics may cause difficulties in drawing conclusions about the frequency of documented pain assessment at the ED. However, documentation is a quality variable and should be considered as a reflection of performed patient care. Another limitation may be the sample size of 120 medical records per year. It may not reflect all documented pain assessment, but the data collection was done over a period of seven years so the result may still reflect the actual frequency of documented pain assessment at the ED.

Since these results were gathered from one urban hospital, the results are not necessarily generalizable to all EDs. However, as we used one commonly used diagnosis that affects all ages of adult patients, other EDs could use the result as a possible quality improvement variable for their own acute care.

The cross-sectional design makes it possible to collect data on one occasion; however, the possibility to bias the data increases over time. Our study design means that we cannot exclude the possibility that other parallel causes, such as educational efforts outside the ED, work load, and staffing may have contributed to an improved pain assessment, and not the described interventions per se.

\section{Conclusion}

Our result shows that mandatory pain assessment in the patient's computerized medical record was successful for a period of time as an intervention to improve the frequencies of documentation of pain assessment during care in the ED. The result was stable for 
21 months, before a slight decline in the frequency of pain assessment was shown. However the re-evaluation of pain scores was nonexistent and the computerized systems must build in a re-evaluation of pain.

\section{References}

Abou-Setta, A.M., Beaupre, L.A., Rashiq, S., Dryden, D.M., Hamm, M.P., Sadowski, C.A., et al., 2011. Comparative effectiveness of pain management interventions for hip fracture: a systematic review. Annals of Internal Medicine. 155 (4), 234-245.

Berben, S.A., Meijs, T.H., van Grunsven, P.M., Schoonhoven, L., van Achterberg, T., 2012. Facilitators and barriers in pain management for trauma patients in the chain of emergency care. Injury. 43 (9), 1397-1402.

Berthier, F., Potel, G., Leconte, P., Touze, M.D., Baron, D., 1998. Comparative study of methods of measuring acute pain intensity in an ED. The American Journal of Emergency Medicine. 16 (2), 132-136.

Bijur, P.E., Latimer, C.T., Gallagher, E.J., 2003. Validation of a verbally administered numerical rating scale of acute pain for use in the emergency department. Academic Emergency Medicine: Official Journal of the Society for Academic Emergency Medicine. 10 (4), 390-392.

Brown, J.C., Klein, E.J., Lewis, C.W., Johnston, B.D., Cummings, P., 2003. Emergency department analgesia for fracture pain. Annals of Emergency Medicine. 42 (2), 197-205.

Cordell, W.H., Keene, K.K., Giles, B.K., Jones, J.B., Jones, J.H., Brizendine, E.J., 2002. The high prevalence of pain in emergency medical care. The American Journal of Emergency Medicine. 20 (3), 165-169.

County Council Stockholm (2006). Focus report on pain (In Swedish: Fokusrapport Smärta). Stockholms Läns Landsting. Stockholm, Sweden.

Court-Brown, C.M., Caesar, B., 2006. Epidemiology of adult fractures: a review. Injury. 37 (8), 691-697.

Decosterd, I., Hugli, O., Tamchès, E., Blanc, C., Mouhsine, E., Givel, J.C., et al., 2007. Oligoanalgesia in the emergency department: short-term beneficial effects of an education program on acute pain. Annals of Emergency Medicine. 50 (4), $462-471$.

Eder, S.C., Sloan, E.P., Todd, K., 2003. Documentation of ED patient pain by nurses and physicians. The American Journal of Emergency Medicine. 21 (4), 253-257.
Fosnocht, D.E., Swanson, E.R., Barton, E.D., 2005. Changing attitudes about pain and pain control in emergency medicine. Emergency Medicine Clinics of North America. 23 (2), 297-306.

Gordon, D.B., Rees, S.M., McCausland, M.R., Pellino, T.A., Sanford-Ring, S. Smith-Helmenstine, J., et al., 2008. Improving reassessment and documentation of pain management. Joint Commission Journal on Quality and Patient Safety. 34 (9), 509-517.

Lewén, H., Gardulf, A., Nilsson, J., 2010. Documented assessments and treatments of patients seeking emergency care because of pain. Scandinavian Journal of Caring Sciences. 24 (4), 764-771.

Motov, S.M., Khan, A.N., 2008. Problems and barriers of pain management in the emergency department: are we ever going to get better? Journal of Pain Research. 2, 5-11.

Niruban, A., Biswas, S., Willicombe, S.C., Myint, P.K., 2010. An audit on assessment and management of pain at the time of acute hospital admission in older people. International Journal of Clinical Practice. 64 (10), 1453-1457.

Purser, L., Warfield, K., Richardson, C., 2014. Making pain visible: an audit and review of documentation to improve the use of pain assessment by implementing pain as the fifth vital sign. Pain Management Nursing. 15 (1), 137-142.

Ritsema, T.S., Kelen, G.D., Pronovost, P.J., Pham, J.C., 2007. The national trend in quality of emergency department pain management for long bone fractures. Academic Emergency Medicine: Official Journal of the Society for Academic Emergency Medicine. 14 (2), 163-169.

Sampson, F.C., Goodacre, S.W., O'Cathain, A., 2014. Interventions to improve the management of pain in emergency departments: systematic review and narrative synthesis. Emergency Medicine Journal. 31 (e1), e9-e18.

Silka, P.A., Roth, M.M., Moreno, G., Merrill, L., Geiderman, J.M., 2004. Pain scores improve analgesic administration patterns for trauma patients in the emergency department. Academic Emergency Medicine: Official Journal of the Society for Academic Emergency Medicine. 11 (3), 264-270.

Tanasale, B., Kits, J., Kluin, P.M., Trip, A., Kluin-Nelemans, H.C., 2013. Pain and anxiety during bone marrow biopsy. Pain Management Nursing. 14 (4), 310-317.

Todd, K.H., Ducharme, J., Choiniere, M., Crandall, C.S., Fosnocht, D.E., Homel, P., et al., 2007. Pain in the emergency department: results of the pain and emergency medicine initiative (PEMI) multicenter study. The Journal of Pain. 8 (6), 460466.

Vazirani, J., Knott, J.C., 2012. Mandatory pain scoring at triage reduces time to analgesia. Annals of Emergency Medicine. 59 (2), 134-138, e132.

Wilson, J.E., Pendleton, J.M., 1989. Oligoanalgesia in the emergency department. The American Journal of Emergency Medicine. 7 (6), 620-623. 